\title{
Assessment of a narrative approach to the diet history
}

\author{
LC Tapsell ${ }^{*}, K$ Pettengell and SL Denmeade \\ Department of Biomedical Science, University of Wollongong, Northfields Avenue, Wollongong, NSW 2522, \\ Australia
}

Submitted 13 February 1998: Accepted 28 September 1998

\begin{abstract}
Objective: To assess the quality of a narrative form diet history (DH).

Design: Reproducibility assessed with data obtained at 6-week intervals. Criterion validity assessed using energy intake to estimated energy expenditure (EI : BMR) cutoff limits. Relative validity assessed by comparing results for energy and macronutrients at baseline and month 2 of an intervention trial with those obtained from 3-day food records (FR).

Setting: Community-based dietary intervention trials for the study of metabolic syndrome in the Illawarra region of New South Wales, Australia.

Subjects: Reproducibility: 43 healthy female volunteers. Mean age 58.72 years (range 50-67), mean body mass index (BMI) 25.79 (range 21-36). Validity: 45 healthy volunteers-18 males (mean age 46.9 years, mean BMI 27.8), and 27 females (mean age 45.7 years, mean BMI 26.2), attending a study on the effect of diet on metabolic variables.

Results: Reproducibility: wide SD values indicated a high degree of intraindividual variation, but correlation coefficients were comparable to those of similar studies. Validity: underreporting was inconsistent with individuals, but was greater with the $\mathrm{DH}$ than the FR at each stage of the dietary trial (significant at month $1, P<0.01$ ). Underreporters indicated higher intakes of protein during the trial $(P<0.05)$. Weaker associations were found between the DH and FR data for energy values, but there was strong agreement for per cent fat consumed as saturated and monounsaturated fat at each stage of the trial.

Conclusions: The narrative form DH performs as well as standardized interviews, but more work needs to be done at the micro level, focusing on aspects which deal with foods likely to be underreported in the particular study context. This can be achieved through continued research using combined methodologies.
\end{abstract}

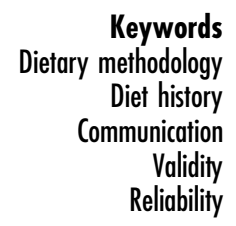

The diet history (DH) has maintained an important position in dietary methodology. The early Burke version comprised an in-depth interview on usual consumption patterns, followed by a food frequency cross-check and a 3-day food record (FR) ${ }^{1}$. Today many versions exist, ranging from open-ended in-depth interviews with trained dietitian/nutritionists to selfadministered questionnaires ${ }^{2}$. The $\mathrm{DH}$ presented here lies in the former category, and follows a rigorous analysis of communication processes in the interview. The rationale for this approach is reported elsewhere , $^{3}$ but whilst the benefits can be argued from a communication perspective, its performance in extracting dietary data warrants evaluation.

Conventions have been developed for validation studies ${ }^{4}$, but these can only be applied once the context of the study has been fully considered 5 . Testing for reproducibility of data from repeat measures is the first step in evaluating a dietary assessment method, but is problematic because both dietary habits and communication processes may change between measures. As reproducibility assessments aim to expose random rather than systematic error, the spread of data and correlations are the statistical features of interest ${ }^{6}$. No gold standard exists amongst dietary intake methods to test validity ${ }^{7}$, although biomedical markers address this need ${ }^{8}$. Doubly labelled water (DLW) studies provide a gold standard for energy estimates but are expensive to use in routine validity assessments. Estimates of energy balance based on DLW studies provide a practical option, with cut-off values for ratios of energy intake to estimated energy expenditure (EI : BMR) providing a means for identifying underreporting in epidemiological studies?. Where the study concerns relationships between current dietary intakes and disease indicators, relative validity can be assessed by comparing data from DH interviews with that of FRs 5 . Deviations between the reference and test method can 
be assessed using a number of statistical approaches including paired and unpaired comparisons, ranking, correlation, regression and Bland Altman analyses, with preference given to the use of measures of dispersion of differences between paired observations (percentage of differences less than $20 \%$ of the mean intake, or SD of the differences), and correlation coefficients (with confidence intervals) as appropriate ways for conducting statistical analyses of validation data $^{6}$. The use of several statistical applications is recommended, particularly in assessing the stability of any relationships exposed through correlation coefficients ${ }^{10}$. With these issues in mind, this article examines the reliability and validity of a narrative form DH in the context of intervention studies focusing on the metabolic syndrome.

\section{Methods}

\section{Context}

The narrative form DH method was appropriate for diet-disease studies in the context described above ${ }^{4}$, particularly given its clinical nature. In keeping with the literature on diet and metabolic syndrome, variables of interest were energy and macronutrient consumption, including a breakdown of fat into polyunsaturated, monounsaturated and saturated fatty acids (PUFA, MUFA, SFA). Reproducibility was examined with healthy volunteers for an ecological study, with no expectations beyond normality during the interval between repeat measures, as would be the case in an intervention study. In contrast, validity was assessed in the context of anticipated use ${ }^{11}$, where unpaid subjects, recruited via local advertisements, were required to report on their eating habits over a number of months. The trial aimed to study the effects of dietary fat on insulin resistance and other biochemical indices. Subjects were randomly assigned to one of three diet types, with specified ratios of dietary fat.

\section{Reproducibility}

\section{Subjects}

Forty-three healthy volunteers provided a detailed DH during spring 1994 and agreed to return in 6 weeks for a repeat interview. The methodological nature of this study did not require a representative sample.

\section{Collection of data}

The interview took $1-1.5 \mathrm{~h}$ and was conducted by three trained dietitians. In view of potential interviewer effects, a different interviewer was used in the second interview. A narrative account of usual, progressive daily intake was requested followed by a food checklist. After the interview, the DH data were converted to a food frequency format by the interviewers using the approach described by Gibson $^{12}$. The dietary data were entered into the Diet 1 nutrient analysis software package (version 4, Xyris Software, Highgate Hill, Brisbane, Australia), which contains the Australian nutrient database NUTTAB (Department of Human Services and Health, Canberra, 1995). Data entry was completed using standardized procedures by a fourth dietitian who was not involved in the interview process.

\section{Statistical analysis}

Mean values for energy and macronutrients were assessed together with the SD of the differences between the two estimations. Correlation coefficients between the first and second estimations were calculated.

\section{Validity}

\section{Subjects}

Following screening for a study on the effect of diet on metabolic variables, 45 healthy volunteers were willing to proceed with the study. Ethical approval was

Table 1 Reproducibility of the narrative form $\mathrm{DH}$ on an aggregate level (mean) and on an individual level (SD of differences) for energy and macronutrient consumption

\begin{tabular}{lrrrrr}
\hline & & & & \multicolumn{2}{c}{ Correlation coefficients } \\
\cline { 3 - 6 } & $\begin{array}{c}\text { Interview 1 } \\
\text { (mean) }\end{array}$ & $\begin{array}{c}\text { Interview 2 } \\
\text { (mean) }\end{array}$ & $\begin{array}{c}\text { SD of } \\
\text { differences }\end{array}$ & $\begin{array}{c}\text { Reported } \\
\text { study }\end{array}$ & $\begin{array}{r}\text { Bittoni and } \\
\text { Wilkens }\end{array}$ \\
\hline Energy (kJ) & 7437.05 & 7489.05 & 1491.41 & 0.79 & 0.70 \\
Protein (\%) & 19.44 & 19.51 & 3.84 & 0.55 & 0.53 \\
CHO (\%) & 48.79 & 48.09 & 7.03 & 0.54 & 0.79 \\
Fat (\%) & 29.00 & 29.74 & 6.80 & 0.70 & 0.67 \\
Alcohol (\%) & 2.81 & 2.62 & 3.09 & 0.66 & \\
Fibre (g) & 29.05 & 29.03 & 8.29 & 0.67 & \\
SFA (\%) & 42.93 & 42.49 & 5.21 & 0.36 & \\
PFA (\%) & 17.90 & 18.28 & 4.72 & & \\
MFA (\%) & 39.14 & 39.33 & & 4.77 &
\end{tabular}

${ }^{*}$ Bittoni and Wilkens $(1994)^{16}$ using a self-administered DH questionnaire.

No significant difference $P>0.05$. 
provided by the University of Wollongong Human Ethics Committee.

\section{Collection of data}

Six student dietitians from the University of Wollongong were responsible for data collection and data entry at baseline and at approximately 1-month intervals during the spring of 1996. Students were trained in the narrative form $\mathrm{DH}$ and regular meetings were attended with supervising dietitians to ensure consistency in approaches. The baseline DH referred to the 3-month period prior to the intervention, and subsequent histories referred to the previous month. They were completed in about $1.5 \mathrm{~h}$. Subjects were instructed on keeping records of 1 weekend day and 2 weekdays representative of usual eating patterns, and to record all food preparation techniques including recipes. Forms were provided for subjects to record by weight, number, measured dimensions or in terms of household measures. Salter Slimmer scales (model 0.36), measuring cups and spoons were provided. Data were managed as for the reproducibility study. Recipes were entered onto the database and a standardized system of data entry was applied across the group.

\section{Statistical analysis}

\section{Criterion validity}

Values for EI and macronutrient consumption was determined for each subject from the DH and FR at baseline and at the end of 1 and 2 months on the programme, respectively. Basal metabolic rate (BMR) was determined using the Schofield equation ${ }^{13}$, and recalculated at each month to allow for slight changes in body weight. Ratios of EI : BMR were then calculated for each subject at each time interval and for each of the dietary methods, and compared with the cut-off limits derived by Goldberg et al. ${ }^{9}$ using the $95 \%$ confidence interval for a physical activity level (PAL) of 1.55. For the $\mathrm{DH}$, the measurement period was 28 days and for the FR 3 days. For individuals the cut-off values were set at 1.14 and 1.06 for the DH and FR, respectively. Subjects with EI : BMR ratios below the cut-off limits were categorized as underreporters, and the frequency of distribution of these subjects throughout the trial were presented. McNemars test for correlated proportions was used to test for significant differences between the number of underreporters identified by both methods of dietary assessment. Logistic regression was applied to test whether underreporting was influenced by method, sex and body mass index (BMI), using BMI categories of 20-25, 26-30 and $>30$. Two sample $t$-tests were applied to determine whether or not there was a significant difference between underreporters and others in terms of their reporting on energy and macronutrients.

\section{Relative validity}

Estimations of energy and macronutrient consumption were examined at baseline and the 2-month stage. The mean and SD of the difference were used to assess the extent of agreement between the two methods at a group and individual level. Related group $t$-tests were used to test for significance $(P<0.05)$. An agreement of at least $80 \%$ calculated as the difference divided by the mean (\%) was chosen as a measure of extent of individual error ${ }^{14}$. Energy and nutrient intakes were defined as sufficiently valid if greater than an arbitrarily calculated $85 \%$ of subjects demonstrated agreement between methods of at least $80 \%$. Correlations between data from the two dietary methods were examined using Pearson's correlation coefficient to detect linear relationships and expressed in terms of confidence intervals as the latter are more informative than a single probability value ${ }^{6}$. Agreement was also assessed by plotting the difference between the two methods against their mean, allowing for investigation of any possible relationship between the measurement error and true value ${ }^{15}$.

\section{Results}

\section{Reproducibility}

The mean age of the 43 female volunteers was 58.72 years (range 50-67 years), with a mean BMI of 25.79 (range 21-36). Table 1 shows the results of both DH interviews for energy and macronutrient consumption. Data for protein, fat and carbohydrate $(\mathrm{CHO})$ consumption are presented as per cent energy and data for SFA, PUFA and MUFA consumption are presented as per cent total fatty acids.

The calculated energy and macronutrient intakes did not differ significantly between the two interviews on an aggregate level. Multiple regression analysis, using the difference as the response variable and age, BMI and mean of the two interviews as the predictor variables also indicated no significant effect on the difference $(P>0.05)$. For each variable, the SD was much higher than the mean difference, indicating that the range was wide, where large positive and negative values cancelled each other out to provide small mean values. The SD for alcohol was particularly high, and there was a tendency to report more fat in the second interview. Correlation coefficients were reasonable. The highest correlation, for energy, was comparable to that found in a larger study from a similar population ${ }^{16}$. This was also the case for the remaining variables presented in the same form for both studies: per cent energy from fat and alcohol, and grams of fibre consumed (see Table 1). The lowest correlation was found for reported intakes of MUFA. 
Table 2 Number of underreporters (UR) stratified by BMI at each stage of the dietary trial ${ }^{a, b}$

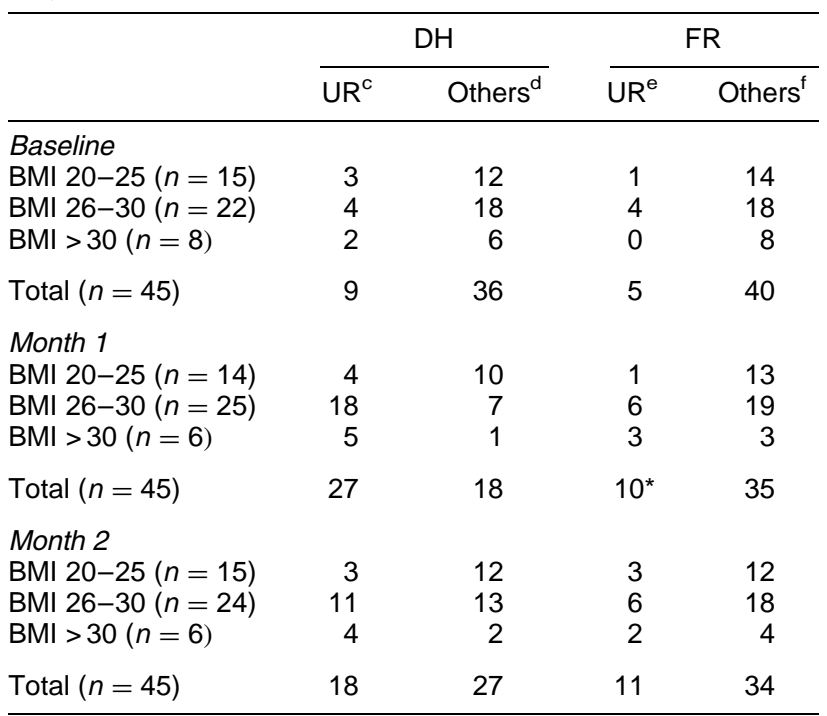

${ }^{*} P<0.01$.

${ }^{a}$ McNemars test for correlated proportions DH vs. FR.

bogistic regression comparing DH and FR with BMI. No significant

relationship between reporter, BMI and method $(P>0.05)$.

CUnder-reporters defined where $\mathrm{El}: \mathrm{BMR}<1.14$.

${ }^{\mathrm{d} O t h e r s ~ d e f i n e d ~ w h e r e ~} \mathrm{El}: \mathrm{BMR}>1.14$

eUnderreporters defined where $\mathrm{El}: \mathrm{BMR}<1.06$.

'Others defined where El : BMR > 1.06.

\section{Criterion validity}

Eighteen males and 27 females participated (mean age $46.9 \pm 6.62$ and $45.7 \pm 8.07$; mean BMI $27.8 \pm 0.05$ and $26.2 \pm 0.06$, respectively). The group were highly educated: over $60 \%$ had tertiary qualifications and only $7 \%$ had not completed school. Sixty-five per cent of the sample worked in managerial, professional or paraprofessional areas, and 20\% were either clerks, salespersons or students. The numbers of underreporters at each stage of the intervention trial for the DH and 3-day FR are given in Table 2. Underreporting was greater with the DH than the FR at each stage throughout the trial and this difference was significant at the 1-month period $(P<0.01)$. There was a greater amount of underreporting during the trial than at baseline. Only four subjects consistently underreported at the three measurement points, in each case via the DH. Although the trend was for overweight people to underreport, and for the $\mathrm{DH}$ to result in more underreporting the differences between subject numbers and methods were not statistically significant $(P>0.05)$.

There was no significant difference between data obtained from underreporters and others in the per cent of energy from macronutrients for either method at baseline $(P>0.05)$, but during the trial underreporters tended to report a higher intake of protein (Table 3 ). This difference was significant at both measurement points for the DH method $(P<0.05)$.

\section{Relative validity}

The differences between the two methods for estimated energy and fat intake were examined in a number of ways (Table 4). The mean differences between energy intakes estimated from each method were significantly different both before and during the trial $(P<0.05)$, but this was not the case for fat intake. While values for the percentage energy from fat was significantly different at baseline $(P<0.05)$, there was no significant difference at month 2 of the trial (although the SD remained relatively high). This was supported by the greater extent of agreement between the two methods for estimated per cent energy from fat. Interestingly, the correlation coefficients (and confidence intervals) for both energy

Table 3 Mean percentage energy contributed by protein, fat, carbohydrate and alcohol at baseline and during the dietary trial for underreporters (UR) and others

\begin{tabular}{|c|c|c|c|c|}
\hline \multirow{2}{*}{$\begin{array}{l}\text { Macronutrient } \\
\text { (\% energy) }\end{array}$} & \multicolumn{2}{|c|}{ Mean (SD) for $\mathrm{DH}^{\mathrm{a}}$} & \multicolumn{2}{|c|}{ Mean (SD) for $\mathrm{FR}^{\mathrm{a}}$} \\
\hline & UR & Others & UR & Others \\
\hline \multicolumn{5}{|l|}{ Baseline } \\
\hline Protein & $19.6(2.83)$ & $18.7(3.02)$ & $19.2(3.42)$ & $18.3(2.71)$ \\
\hline Fat & $28.9(4.70)$ & $33(5.83)$ & $30.4(7.3)$ & $33.6(6.92)$ \\
\hline Carbohydrate & $47.0(6.59)$ & $45.6(6.13)$ & $46.8(4.76)$ & $44.8(7.51)$ \\
\hline Alcohol & $4.6(4.25)$ & $2.7(3.45)$ & $3.6(5.36)$ & $3.4(3.71)$ \\
\hline \multicolumn{5}{|l|}{ Month 1} \\
\hline Protein & $20.2(2.62)$ & $18.1(4.13)^{\star}$ & $19.1(3.73)$ & $18.8(2.71)$ \\
\hline Fat & 30.7 (5.52) & $33.2(6.90)$ & $34(2.74)$ & $33.1(5.65)$ \\
\hline Carbohydrate & $45.7(5.34)$ & $46.9(6.17)$ & $44.1(5.20)$ & $45.4(5.37)$ \\
\hline Alcohol & $3.4(3.69)$ & $1.8(2.31)$ & $2.8(4.13)$ & $2.6(2.97)$ \\
\hline \multicolumn{5}{|l|}{ Month 2} \\
\hline Protein & $20.2(2.94)$ & $18.37(2.69)^{*}$ & $17.7(2.76)$ & $17.9(3.09)$ \\
\hline Fat & $35.2(4.85)$ & $34.3(4.67)$ & 34.5 (5.79) & $34.9(6.12)$ \\
\hline Carbohydrate & $42.9(4.91)$ & $45(4.94)$ & $46.1(6.04)$ & $44(5.71)$ \\
\hline Alcohol & $1.7(2.60)$ & $3.0(5.19)$ & $1.6(3.26)$ & $3.1(3.50)$ \\
\hline
\end{tabular}

aTwo sample $t$-tests.

${ }^{\star} P<0.05$. 
Table 4 Mean differences, extent of agreement and correlation coefficients for energy and fat consumption data obtained from DHs and FRs at baseline and month 2 of a dietary trial

\begin{tabular}{|c|c|c|c|c|c|c|}
\hline & \multicolumn{2}{|c|}{$\begin{array}{l}\text { Mean differences } \\
\text { DH-FR (SD) }\end{array}$} & \multicolumn{2}{|c|}{$\begin{array}{l}\text { Number with over } \\
80 \% \text { agreement }\end{array}$} & \multicolumn{2}{|c|}{$\begin{array}{c}\text { Correlation } \\
\text { coefficients }(\mathrm{Cl})^{\mathrm{c}}\end{array}$} \\
\hline & Baseline & Month 2 & Baseline & Month 2 & Baseline & Month 2 \\
\hline Energy (kJ) & $-891(1604)^{\star \star}$ & $-695(1513)^{\star *}$ & 31 & 32 & $0.70(0.51-0.82)^{*}$ & $0.70(0.51-0.82)^{*}$ \\
\hline Fat (\%E) & $-1.6(5.4)^{\star \star}$ & $-0.1(4.7)$ & 36 & $40 \dagger$ & $0.64(0.43-0.78)^{*}$ & $0.65(0.44-0.79)^{\star}$ \\
\hline PFA (\%E) & $0.3(4.6)$ & $0.2(3.4)$ & 28 & 31 & $0.73(0.56-0.86)^{*}$ & $0.75(0.59-0.85)^{*}$ \\
\hline MFA (\%E) & $0.3(4.5)$ & $0.0(3.4)$ & $41 \dagger$ & $45 \dagger$ & $0.47(0.21-0.69)^{*}$ & $0.96(0.93-0.98)^{*}$ \\
\hline SFA (\%E) & $-0.3(5.0)$ & $-0.3(4.6)$ & $43 \dagger$ & $41 \dagger$ & $0.75(0.59-0.85)^{*}$ & $0.91(0.84-0.95)^{*}$ \\
\hline
\end{tabular}

${ }^{*} P<0.01$.

${ }^{* *} P<0.05$.

$\dagger>85 \%$ subjects had $>80 \%$ agreement between methods.

a Related group $t$-tests.

${ }^{\mathrm{b}}$ Extent of agreement.

'Pearson's correlation coefficient.

and fat remained much the same for baseline and trial measurements. Mean differences in fatty acid values were not significantly different for either baseline or trial measurements, but the extent of agreement for MUFA and SFA values was greater than that for PUFA values. In addition, while the correlation coefficients (and confidence intervals) remained much the same for PUFA values, as they did for fat and energy, they improved substantially for SFA and MUFA values during the trial period. Improved clustering around zero in the Bland-Altman plots for MUFA values from the trial period supported this observation (Fig. 1).

\section{Discussion}

\section{Reliability}

One of the major problems in comparing studies of the $\mathrm{DH}$ is that it is not a singular method, rather it represents a wide range of approaches which focus on an account of usual eating patterns. Despite this, a review of repeatability studies on DHs (as a whole) has demonstrated the ability of the method to produce reliable group means for energy and macronutrients, although the findings are somewhat variable ${ }^{17}$. Care needs to be taken in comparing the results from different populations, with due attention to contextual

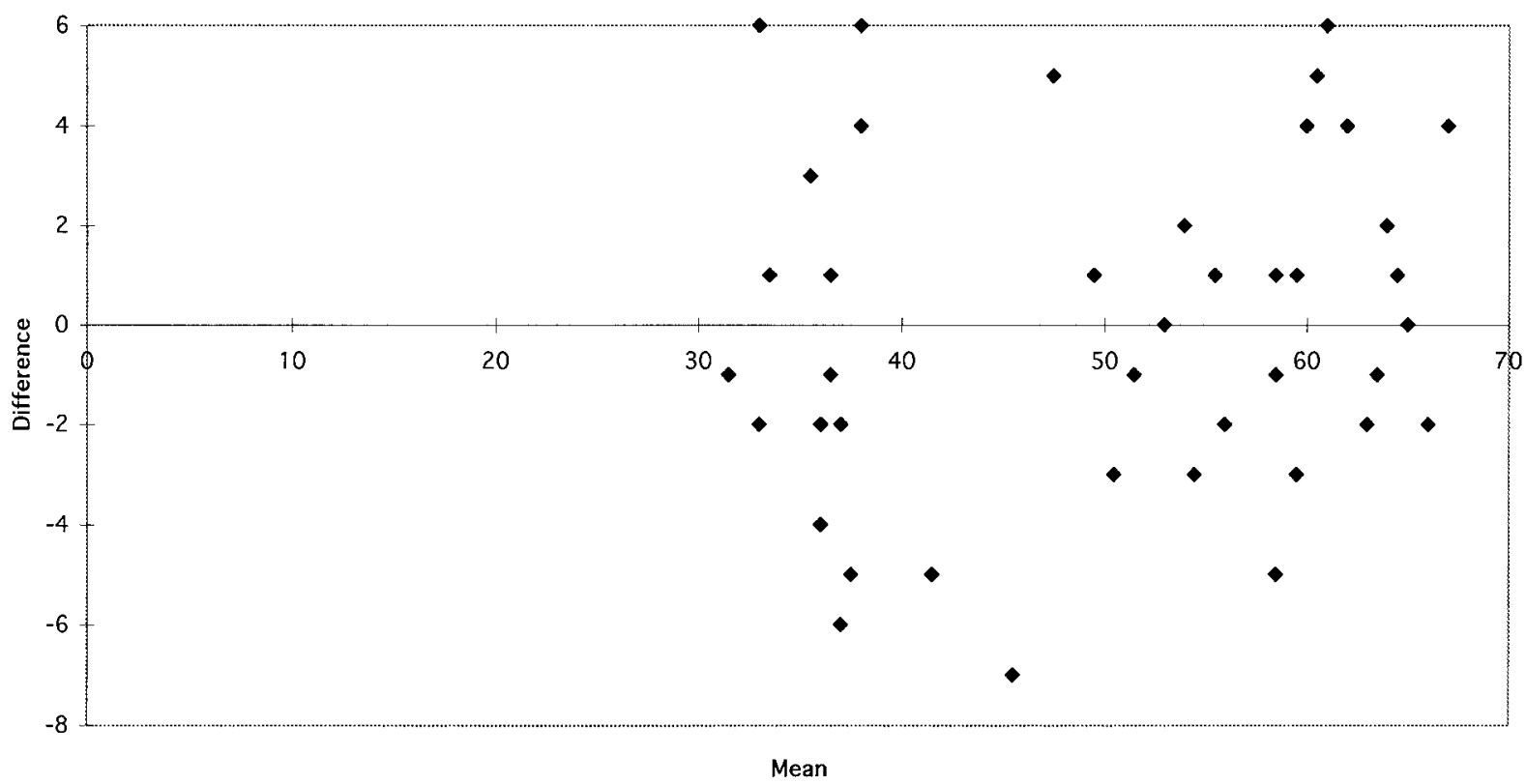

Fig. 1 Difference (DH-FR) plotted against mean for MUFA intake at 2 months intervention 
variations. In the study reported here, correlation coefficients compared well with those from a larger study of mid-aged Western women, but in the latter case the DH was a self-administered questionnaire ${ }^{16}$. This suggests that, at the group level, the narrative interview strategy is as reliable as a standardized questionnaire, and that whether the method leans more towards an open or closed schedule, the concept of usual eating patterns is somehow tangible. The tendency observed in our study to report higher intakes of fat in the repeat interview suggests the effect described by Hebert et $a l .^{18}$ where social desirability may be less evident in repeat measures as reactivity is reduced by increased exposure. The low correlation coefficients and large data spread observed in our study for values of MUFA intake may be linked to the variation in values for PUFA intakes. PUFA- and MUFAbased oils and margarines compete for positions in the diet and, where particular products are identified, there may be a temporal effect on the reporting of their usual consumption. Variability at the individual level implied further work is needed on how specific food items are reported, in particular fat-rich foods and alcohol.

\section{Validity}

Using estimates of EI:BMR, the number of underreporters was found to be greater during the intervention than at baseline, and with the $\mathrm{DH}$ method than the FR at each measurement point. The first finding may well reflect dieting, a phenomenon experienced with intervention trials ${ }^{19}$ even though, as in our case, weight maintenance was advised. The second finding is less easily explained, at least at the baseline estimate. Whilst estimates of EI : BMR provide reasonable assessments of plausibility, there may be problems with the use of cut-off points at the individual level, particularly where inadequate attention has been be paid to activity factors and estimates of $\mathrm{BMR}^{20}$. Also, the intervention itself may affect reporting. In a multicentre trial, compliance was found to be overestimated with food records and better estimated by unannounced 24-h recalls ${ }^{21}$ and in a smaller intervention trial the lack of reporting of foods high in fat was attributed to the provision of dietary information ${ }^{22}$. In the latter study underreporting was thought to be due to concealment of foods containing fat and sugar, or not belonging to meals, given their relative absence in records. This may support our finding that underreporters tended to report a relatively higher intake of protein during the trial, a trend discussed elsewhere in the literature ${ }^{20}$. Underreporting was not consistent and it did not appear related to the sex of the subjects, their BMI or the method of dietary assessment, although this may reflect the small numbers in our study. The literature is also ambiguous in this regard particularly as to whether or not overweight people systematically underreport and normal weight people do not. In fact there may be real problems in trying to generalize about this phenomenon using standard categorizations of population subgroups (see for example Macintyre and Anderson $^{23}$ ), with the answers more likely to lie in the context of the study. Apart from issues concerning physiological measurements as standards, our study confirms the observation by Mertz et al. ${ }^{24}$ that underreporting does not appear to have a degree or direction for different people and the data provide very few indications of the nature of underreporting. It would appear that the narrative form $\mathrm{DH}$ is not free of this dimension and that further investigations into why this might be so are warranted. Research into psychosocial aspects of data collection is now an acknowledged priority area for dietary methodology ${ }^{25}$, with a focus on current understandings of communication processes ${ }^{26}$.

Relative validity data at baseline and 2 months into the dietary trial confirm the notion that social factors (i.e. the context of the study) impinge on outcomes. A study in the UK found that reductions in SFA were achievable in a community-based trial, but that increases in MUFA were difficult given the small range of foods available to meet this goal ${ }^{27}$. It may be that the narrower confidence interval found for MUFA in our intervention trial reflected this narrow choice of foods with which subjects soon became familiar, and the improvements in reporting on MUFA may have helped to improve the values for total fat.

\section{Future directions}

This study has demonstrated that data from the narrative form $\mathrm{DH}$ is reliable and valid in communitybased studies examining energy and macronutrient consumption. The techniques implicit in this framework have appeared in other studies where the $\mathrm{DH}$ methods incorporated conversational features ${ }^{26}$. Future developments of the narrative form DH would need to move beyond the basic framework provided by the narrative structure to a specific focus on foods known to be underreported (such as how and when to ask about them). Identification of these aspects would provide a start to displaying how problematic certain topics become, and how they might be more successfully dealt with. Research in similar areas also provides direction ${ }^{28}$.

There is still a long way to go and it will not be easy. Combining methodologies is noble and exciting, but it is also fraught with difficulties, particularly where basic assumptions appear at first incompatible. Common ground can be found in the recognition that error is inherent in measurement ${ }^{29}$, or put another way, there is no 'context free' measurement ${ }^{30}$. As Barrett-Connor ${ }^{31}$ argues, excessive certainty regarding the value of a method is not important, what is important is the 
careful consideration of results. Theoretical positions are important, and this includes an understanding of context. In dietary methodology we need to know what works best in a given situation ${ }^{32}$. All the better if we can demonstrate the fact and explain why this is so.

\section{Acknowledgements}

We would like to acknowledge the co-operation of volunteers for these studies, and the research team: Ruth Nielson, Margaret Wilson, Nicole Senior, Marie Martin, Tanya Hazelwood, Corinne White, Dane Crandon, Sharmala Ratnesar, Joanne Scilazi, Sheena McGhee, Elaine Knight, Dr Barbara Meyer and Professor Dennis Calvert, for their parts in data collection and management, Dr Ken Russell for statistical advice and Professor Len Storlien for support throughout the study and review of the manuscript.

\section{References}

1 Burke BS. The dietary history as a tool in research. J. Am. Diet. Assoc. 1947; 23: 1041-46.

2 Pao EM, Cypel YS. Estimation of dietary intake. In: Ziegler E, Filer LJ, eds. Present Knowledge in Nutrition. Washington DC: ILSI Press, 1996: 498-508.

3 Tapsell LC. The dietetic interview: towards a critical social pedagogy. PhD thesis, Wollongong: University of Wollongong, 1995.

4 Block G, Hartman AM. Issues in reproducibility and validity of dietary studies. Am. J. Clin. Nutr. 1989; 50: 113-8.

5 Nelson M. The validation of dietary assessment. In: Margetts BM, Nelson M, eds. Design Concepts in Nutritional Epidemiology, second edition. Oxford: Oxford University Press, 1997: 241-72.

6 Burema J, van Staveren W, Feunekes GIJ. Letter to the editor: guidelines for reports on validation studies. Eur. J. Clin. Nutr. 1995; 49: 932-3.

7 Mertz W. Food intake measurements: is there a 'gold standard'? J. Am. Diet. Assoc. 1992; 92: 1463-5.

8 Bingham S, Day N. Using biochemical markers to assess the validity of prospective dietary assessment methods and the effect of energy adjustment. Am. J. Clin. Nutr. 1997; 65 (suppl): 1130S-1137S.

9 Goldberg GR, Black AE, Jebb SA et al. Critical evaluation of energy intake data using fundamental principles of energy physiology. 1. Derivation of cut-off limits to identify underrecording. Eur. J. Clin. Nutr. 1991; 45: 569-81.

10 Bellach B. Remarks on the use of Pearson's correlation coefficient and other association measures in assessing validity and reliability of dietary assessment methods. Eur. J. Clin. Nutr. 1993; 47 (suppl): S42-S45.

11 Kristal AR, Beresford SA, Lazovich D. Assessing change in diet-intervention research. Am. J. Clin. Nutr. 1994; 59 (suppl): 185S-189S.

12 Gibson RS. Qualitative dietary assessment. In: Gibson RS, Nutritional Assessment: a Laboratory Manual. Oxford: Oxford University Press, 1993: 15-22.

13 Schofield WN. Predicting basal metabolic rate. New standards and review of previous work. Hum. Nutr. Clin. Nutr. 1985; 39 (suppl 1): 5-41.

14 Mahalko JK, Johnson LK, Gallagher SK, Milne DB. Comparison of diet histories and seven day food records in a nutritional assessment of older adults. Am. J. Clin. Nutr. 1985; 42 542-53.

15 Bland JM, Altman DG. Statistical methods for assessing agreement between two methods of clinical measurement. Lancet 1986; 1: 307-10.

16 Bittoni MA, Wilkens JR. Assessment of the reliability of a diet history questionnaire. Nutr. Cancer 1994; 21: 143-55.

17 Nelson M, Bingham SA. Assessment of food consumption and nutrient intake. In: Margetts BM and Nelson M, eds. Design Concepts in Nutritional Epidemiology, second edition. Oxford: Oxford University Press, 1997: 123-69.

18 Hebert JL, Clemow L, Pobert L, Ockene IS, Ockene JK. Social desirability bias in dietary self-report may compromise validity intake measures. Int.J. Epidemiol. 1995; 24: 389-98.

19 Morgan S, O'Dea K, Sinclair AL. A low-fat diet supplemented with monounsaturated fat results in less HDL-C lowering than a very-low-fat diet. J. Am. Diet. Assoc. 1997; 97: 151-56.

20 Black AS, Bingham S et al. Validation of dietary intakes of protein and energy against 24 hour urinary $\mathrm{N}$ and DLW energy expenditure in middle-aged women, retired men and post-obese subjects: comparisons with validation against presumed energy requirements. Eur. J. Clin. Nutr. 1997; 51: 405-13.

21 Buzzard IM, Faucett CL, Jeffrey RW. Monitoring dietary change in a low fat diet intervention study: advantages of using 24-h dietary recalls vs food records. J. Am. Diet. Assoc. 1996; 60: 574-9.

22 Grace C, Summerbell C. Does provision of additional dietary information affect actual or only reported compliance to a low-fat diet over 12 weeks in hyperlipidaemic individuals? Report of a pilot study. J. Hum. Nutr. Diet. 1996; 9: 303-7.

23 Macintyre S, Anderson A. Socio-demographic and psychosocial variables. In: Margetts BM, Nelson M, eds. Design Concepts in Nutritional Epidemiology, second edition. Oxford: Oxford University Press, 1997: 273-88.

24 Mertz W, Tsui JC, Judd JT. What are people really eating? The relation between energy intake derived from estimated diet records and intake determined to maintain body weight. Am. J. Clin. Nutr. 1991; 54: 291-5.

25 Buzzard IM, Sievert YA. Research priorities and recommendations for dietary assessment methodology. Am. J. Clin. Nutr. 1994; 59 (suppl): 275S-80S

26 Kohlmeier L. Gaps in dietary assessment methodology: meal- vs list-based methods. Am. J. Clin. Nutr. 1994: 59 (suppl): 175S-179S.

27 Knapper J, Tredger J, Webb D. Substitution of dietary monounsaturated acids for saturated fatty acids in a freeliving population: a feasibility study. J. Hum. Nutr. Diet. 1996; 9: 273-82.

28 Means B, Swan GE, Jobe JB, Esposito JL. An alternative approach to obtaining personal history data. In: Biemer PP, Groves RM et al., eds. Measurement Errors in Surveys. New York: Wiley Interscience, 1991: 167-84.

29 Beaton GH. Approaches to analysis of dietary data: relationship between planned analyses and choice of methodology. Am. J. Clin. Nutr. 1994; 59 (suppl): 253S-61S.

30 Schaeffer NC. Conversation with a purpose-or conversation? Interaction in the standardized interview. In: Biemer PP, Groves RM et al., eds. Measurement Errors in Surveys. New York: Wiley Interscience, 1991: 367-92.

31 Barrett-Connor, E. Nutrition epidemiology: how do we know what they ate? Am. J. Clin. Nutr. 1991; 54 (suppl): 182S-187S

32 Cassidy CM. Walk a mile in my shoes: culturally sensitive food-habit research. Am. J. Clin. Nutr. 1994; 59 (suppl): 190S-197S. 


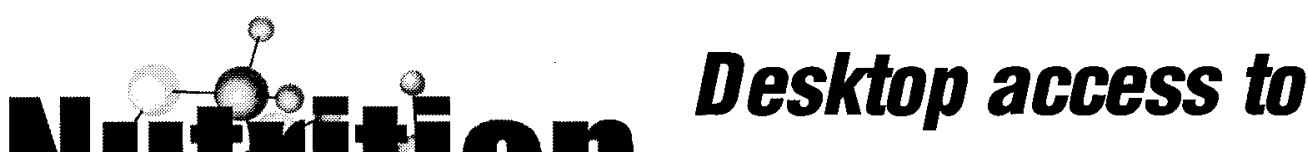 Ninstion worldwide nutritional carbwe $b$ science information}

\section{Access these journals on the Internet:}

- British Journal of Nutrition

- $\quad$ Nutrition Research Reviews

- $\quad$ Proceedings of the Nutrition Society

- Public Health Nutrition

- Nutrition Abstracts and Reviews Series A: Human and Experimental

- Nutrition Abstracts and Reviews Series B: Livestock Feeds and Feeding

Access to the electronic version of each journal is FREE to members of institutions/organizations with a current print subscription to that journal. All you have to do is register.

For more information about this unrivalled resource in nutritional science information and for details of how to register, visit Nutrition CABWeb now at:

\section{http://nutrition.cabweb.org}

(11) CABI Publishing

$\mathrm{CAB}$ International

Wallingford

Oxon, OX10 8DE, UK

Tel: $+44(0) 1491832111$

Fax: $+44(0) 1491829198$

Email: publishing@cabi.org
(11) CABI Publishing

CAB International

10 East 40th Street, Suite 3203

New York, NY 10016, USA

Tel: +12124817018

Fax: +12126867993

Email: cabi-nao@cabi.org 